\title{
EFEITOS DA ACUPUNTURA NA FIBROMIALGIA: UMA REVISÃO BIBLIOMÉTRICA
}

\author{
Rafael da Silva PASSOS*, Sara Souza Vilas BÔAS \& Rahab Vieira PRATES \\ Universidade Estadual do Sudoeste da Bahia, Vitória da Conquista, BA, Brasil. \\ *Autor para correspondência: rafaelspassos08@gmail.com \\ http://dx.doi.org/10.18571/acbm.106
}

\section{RESUMO}

Fibromialgia (FM) é definida como dor musculoesquelética generalizada e crônica com sensibilidade dolorosa em pelo menos 11 dos 18 tender points. A acupuntura é uma das terapêuticas da Medicina Tradicional Chinesa que objetiva diagnosticar doenças e promover a cura pela estimulação da força de autocura do corpo. A partir dessa perspectiva, a acupuntura surge como uma ferramenta útil para controle dos sintomas da FM. Foi realizada uma revisão bibliométrica, a partir de um levantamento de artigos científicos na base de dados BVS, e foram encontrados 18 artigos relevantes para o estudo, por abordar a etiologia e o tratamento da fibromialgia, com foco na acupuntura. A acupuntura apresenta efeitos significativamente positivos sobre qualidade de vida, sono, rigidez, ansiedade, depressão e dor dos pacientes fibromiálgicos, sendo que tais efeitos também se manifestam sistemicamente, através de melhora da dor de outros pontos que não os que foram tratados. A melhora observada nesses pacientes é provocada pela liberação de endorfinas a nível central, diminuindo o nível da dor e a quantidade de serotonina nas plaquetas. A acupuntura é eficaz no tratamento da fibromialgia, mas ainda são necessários mais estudos para maior comprovação dos efeitos listados.

Palavras chave: Fibromialgia; Acupuntura; Fisioterapia.

\begin{abstract}
Fibromyalgia (FM) is defined as generalized musculoskeletal and chronic pain with tenderness in at least 11 of 18 tender points. Acupuncture is one of the therapies of Traditional Chinese Medicine which aims to diagnose diseases and promote healing by stimulating the body self-healing power. From this perspective, acupuncture appears as a useful tool for controlling the symptoms of FM. This is a bibliometric review study, being conducted a survey of scientific papers in the BVS database, and found 18 relevant articles to the study, by addressing the etiology and the treatment of fibromyalgia, focusing on acupuncture. Acupuncture has significantly positive effects on quality of life, sleep, stiffness, anxiety, depression and pain of fibromyalgia patients, and these effects also manifest systemically through pain relief from other points than those that were treated. The improvement observed in these patients is caused by the release of endorphins to the central level, reducing the level of pain and the amount of serotonin in platelets. Acupuncture is effective in treating fibromyalgia, but we still need more studies to further confirmation of the listed effects.
\end{abstract}

Keywords: Fibromyalgia; Acupuncture; Physiotherapy. 


\section{Introdução}

Fibromialgia (FM) é definida como dor musculoesquelética generalizada e crônica com sensibilidade dolorosa em pelo menos 11 dos 18 tender points, de caráter não inflamatório. Depressão, ansiedade e distúrbios do sono são indicados como sintomas frequentes da fibromialgia, que acabam por gerar um grande impacto na qualidade de vida dos pacientes. A referida patologia só é considerada uma síndrome por ser identificada, sobretudo pelo número de sintomas, e não por uma função específica. Não é necessário que o paciente apresente todos os sintomas para que o diagnóstico seja fechado (HEYMANN et al, 2010).

Fibromialgia é uma síndrome reumática que ocorre, sobretudo em mulheres com idade entre 40 e 55 anos (Santos et al 2006), sua etiologia ainda é desconhecida, sendo considerada por alguns autores como uma síndrome de somatização. Porém a partir da década de 1980, passou a ser entendida como uma síndrome de dor crônica na qual a sensibilidade do Sistema Nervoso Central (SNC) à dor apresenta o principal papel (STIVAL et al, 2014). Estudo comprava também a presença da patologia entre crianças, sua prevalência na faixa etária pediátrica é de 1,2 a $6,2 \%$ (DIAS et al, 2012).

Segundo Stivial et al (2014) a qualidade de vida dos pacientes com fibromialgia em idade produtiva de trabalho está diminuída, pois, além da dor, a fadiga e a fraqueza subjetiva levam a incapacidade para o trabalho, o que provoca diminuição de renda familiar. Dessa forma pessoas que sofrem com a FM geralmente procuram pela medicina alternativa e complementar para seu tratamento, sendo a acupuntura um dos recursos mais utilizados (BARBOSA et al, 2013).

A acupuntura é uma das terapêuticas da Medicina Tradicional Chinesa que objetiva diagnosticar doenças e promover a cura pela estimulação da força de autocura do corpo, o que é feito através do realinhamento e redirecionamento da energia, com inserção de agulhas em pontos específicos do corpo, os acupontos, que também podem ser estimulados com calor, pressão, ultrassom, eletricidade e laser (KUREBAYASHI, 2009). Seus efeitos neurobiológicos, que interferem sobre os neurotransmissores relacionados à dor e à depressão, qualificam a técnica como adequada para o tratamento da dor crônica.

A Acupuntura tem sido bastante estudada no Ocidente e aceita pela medicina ocidental como um recurso terapêutico para vários transtornos, especialmente os dolorosos, sendo recomendada pela Organização Mundial de Saúde 2000, para o tratamento de várias doenças. Dentro desse contexto, a FM pode ser explicada tanto pela teoria dos cinco elementos e dos Zang e Fu (órgãos Yin e Yang), quanto pela teoria dos meridianos. Segundo a teoria dos cinco elementos, as pessoas com fibromialgia apresentam transtorno energético no fígado, baçopâncreas e rim. O excesso de energia do fígado leva ao aparecimento dos distúrbios do sono, que se associa aos sintomas de dor crônica no sistema musculoesquelético (BARBOSA et al, 2013).

Cristo (2006) indicou que a fibromialgia é a segunda afecção reumatológica mais frequente, superada apenas pela osteoartrite como causa de dor musculo-esquelética crônica. Nessa mesma pesquisa indica-se uma forte predominância do sexo feminino (80-90\%), uma incidência aumentada na faixa entre 30 e 50 anos e que o risco aumenta significativamente em parentes consanguíneos de pacientes com FM.

O tratamento da FM, recomendado pela Sociedade Brasileira de Reumatologia, é paliativo, voltado apenas para a melhora dos sintomas, sem cura definitiva da síndrome. São utilizados meios farmacológicos como o uso de relaxante e sedativo, melhorando a dor, os transtornos do sono e humor; bloqueadores da recaptação de serotonina, com efeito analgésico periférico e central; uso de benzodiazepínicos, para os transtornos de sono e também os não farmacológicos como a realização de exercícios aeróbicos de baixo impacto, como dança, hidroginástica, natação ou caminhada, realizados três vezes por semana; é importante salientar a necessidade de suporte psicológico, devido aos distúrbios psiquiátricos; e além disso os tratamentos alternativos, como acupuntura e hipnose (PROVENZA et al, 2004) 
A partir dessa perspectiva, a acupuntura surge como uma ferramenta útil para controle dos sintomas da FM. Deste modo, esse estudo visa avaliar os efeitos da acupuntura na fibromialgia a partir de uma revisão da literatura.

\section{Materiais e Métodos}

Trata-se de uma análise bibliométrica, onde foi realizado um levantamento de artigos científicos, na base de dados BVS. A busca foi conduzida utilizando as palavras-chave: Fibromialgia, Acupuntura, sendo realizada a busca em setembro de 2016. Foram selecionados 18 trabalhos, uma vez que abordavam a etiologia e o tratamento da fibromialgia, entre eles a acupuntura.

\section{Resultados}

Até setembro de 2016, foram encontrados 19209 artigos sobre acupuntura, 7113 sobre fibromialgia e 98 sobre Fibromialgia e Acupuntura, que foi o foco do trabalho. O quadro 1 traz detalhes sobre a busca individual de cada termo.

Quadro 1. Pesquisa individual dos termos.

\begin{tabular}{|l|c|}
\hline Filtros utilizados & Número de artigos \\
\hline Acupuntura & 19.209 \\
\hline Texto completo & 4.603 \\
\hline Humanos & 3.841 \\
\hline Ano 2006-2016 & 3.442 \\
\hline Assunto (analgesia por acupuntura) & 171 \\
\hline Termo utilizado & 7.113 \\
\hline Fibromialgia & 3.071 \\
\hline Texto completo & 2.861 \\
\hline Humanos & 2.711 \\
\hline Ano 2006-2016 & 66 \\
\hline Assunto (manejo da dor) & \\
\hline
\end{tabular}

No quadro 2, são dados detalhes sobre a busca conjugada dos termos Acupuntura AND Fibromialgia, bem como os filtros utilizados para melhor refino dos artigos.

Quadro 2: Pesquisa conjugada dos termos

\begin{tabular}{|l|c|}
\hline Filtros utilizados & Número de artigos \\
\hline Acupuntura AND fibromialgia & 98 \\
\hline Acupuntura AND fibromialgia + em humanos & 88 \\
\hline Acupuntura AND fibromialgia + em humanos + Ano 2006 - 2016 & 53 \\
\hline $\begin{array}{l}\text { Acupuntura AND fibromialgia + em humanos + Ano 2006 - 2016 + Assuntos } \\
\text { (Fibromialgia; terapia por acupuntura; dor; analgesia por acupuntura; manejo da dor; } \\
\text { medicina tradicional chinesa) }\end{array}$ & \\
\hline $\begin{array}{l}\text { Acupuntura AND fibromialgia + em humanos + Ano 2006 - 2016 + Assuntos } \\
\text { (Fibromialgia; terapia por acupuntura; dor; analgesia por acupuntura; manejo da dor; } \\
\text { medicina tradicional chinesa) + completo }\end{array}$ & 18 \\
\hline
\end{tabular}

No quadro 3, são verificados os países de publicação referentes aos termos isolados Acupuntura e Fibromialgia, bem como a junção de ambos os termos, contendo o respectivo número de artigos por país. 
Quadro 3: Principais países em número de publicações utilizando os termos fibromialgia, acupuntura e Acupuntura AND Fibromialgia.

\begin{tabular}{|c|c|c|c|c|c|}
\hline \multicolumn{2}{|c|}{ Fibromialgia } & \multicolumn{2}{c|}{ Acupuntura } & \multicolumn{2}{c|}{ Fibromialgia AND Acupuntura } \\
\hline País & $\mathbf{N}^{\mathbf{0}}$ artigos & País & $\mathbf{N}^{\mathbf{0}}$ artigos & País & $\mathbf{N}^{\mathbf{0}}$ artigos \\
\hline EUA & 1527 & China & 3461 & EUA & 19 \\
\hline Brasil & 388 & EUA & 1201 & Brasil & 14 \\
\hline Espanha & 335 & Reino Unido & 509 & China & 9 \\
\hline Canadá & 244 & Brasil & 393 & Suécia & 5 \\
\hline Alemanha & 238 & Alemanha & 262 & Reino Unido & 4 \\
\hline Itália & 210 & Japão & 260 & Espanha & 3 \\
\hline Turquia & 202 & Suécia & 185 & Alemanha & 2 \\
\hline Suécia & 197 & Taiwan & 181 & Itália & 2 \\
\hline Reino Unido & 171 & Coreia do Sul & 180 & Turquia & 2 \\
\hline Israel & 132 & Austrália & 159 & Chile & 1 \\
\hline
\end{tabular}

No quadro 4, estão contidas informações sobre as principais revistas com publicações sobre Fibromialgia AND Acupuntura.

Quadro 4: Principais revistas em número de publicações utilizando os termos Acupuntura AND Fibromialgia.

\begin{tabular}{|l|c|}
\hline Revistas & $\mathbf{N}^{\mathbf{0}}$ artigos \\
\hline Zhongguo Zhen Jiu & 6 \\
\hline Acupunct Med & 5 \\
\hline Altern Ther Health Med & 3 \\
\hline Ann Intern Med & 3 \\
\hline J Altern Complement Med & 3 \\
\hline Rheumatology (Oxford) & 3 \\
\hline Baillieres Best Pract Res Clin Rheumatol & 2 \\
\hline Best Pract Res Clin Rheumatol & 2 \\
\hline Clin Exp Rheumatol & 2 \\
\hline Curr Pharm Des & 2 \\
\hline
\end{tabular}

No quadro 5, estão listados os artigos encontrados na base de dados BvS utilizando os filtros supracitados, que foram utilizados nesse trabalho. 


\section{Biomedica Brasiliensia}

Quadro 5: Artigos filtrados.

\section{Referências}

Short-term complementary and alternative medicine on quality of life in women with fibromyalgia. Dias, Paulo Araujo; Guimarães, André Brito Bastos; Albuquerque, Andrea de Oliveira; de Oliveira, Karoline Lucas; Cavalcante, Maria Luzete Costa; Guimarães, Sergio Botelho. J Integr Med; 14(1): 29-35, 2016 Jan.

Using a partially randomized patient preference study design to evaluate the therapeutic effect of acupuncture and cupping therapy for fibromyalgia: study protocol for a partially randomized controlled trial. Cao, Hui-Juan; Liu, Jian-Ping; Hu, Hui; Wang, Nissi S. Trials; 15: 280, 2014.

Abdominal acupuncture changes cortical responses to nociceptive stimuli in fibromyalgia patients.

de Tommaso, Marina; Delussi, Marianna; Ricci, Katia; D'Angelo, Gemma.

CNS Neurosci Ther; 20(6): 565-7, 2014 Jun.

Short-term improvement following dry needle stimulation of tender points in fibromyalgia.Casanueva, Benigno; Rivas, Paula; Rodero, Baltasar; Quintial, Covadonga; Llorca, Javier; González-Gay, Miguel A. Rheumatol Int; 34(6): 861-6, 2014 Jun.

Acupuncture for treating fibromyalgia. Deare, John C; Zheng, Zhen; Xue, Charlie C L; Liu, Jian Ping; Shang, Jingsheng; Scott, Sean W; Littlejohn, Geoff.

Cochrane Database Syst Rev; (5): CD007070, 2013.

Effect of acupuncture at tender points for the management of fibromyalgia syndrome: a case series. Bastos, Jessica Lucia Neves; Pires, Elisa Dória; Silva, Marcelo Lourenço; de Araújo, Fernanda Lopes Buiatti; Silva, Josie Resende Torres. J Acupunct Meridian Stud; 6(3): 163-8, 2013 Jun.

An overview of systematic reviews of complementary and alternative medicine for fibromyalgia.Terry, Rohini; Perry, Rachel; Ernst, Edzard. Clin Rheumatol; 31(1): 55-66, 2012 Jan.

Acupuntura em adolescentes com fibromialgia juvenil / Acupuncture in adolescents with juvenile fibromyalgia / Dias, Marialda Hõfling P; Amaral, Elisabete; Pai, Hong Jin; Tsai, Daniela Terumi Y; Lotito, Ana Paola N; Leone, Claudio; Silva, Clovis Artur.Rev Paul Pediatr; 30(1): 6-12, 2012. tab.

Traditional Chinese medicine diagnoses in a sample of women with fibromyalgia. Mist, Scott D; Wright, Cheryl L; Jones, Kim Dupree; Carson, James W. Acupunct Med; 29(4): 266-9, 2011 Dec.

Effects of acupuncture on patients with fibromyalgia: study protocol of a multicentre randomized controlled trial. Vas, Jorge; Modesto, Manuela; Aguilar, Inmaculada; Santos-Rey, Koldo; Benítez-Parejo, Nicolás; Rivas-Ruiz, Francisco. Trials; 12: 59, 2011.

Comment on: Efficacy of acupuncture in fibromyalgia syndrome--a systematic review with a meta-analysis of controlled clinical trials. Ernst, Edzard. Rheumatology (Oxford); 49(7): 1420-1; author reply 1421, 2010 Jul.

Traditional Chinese Medicine for treatment of fibromyalgia: a systematic review of randomized controlled trials. Cao, Huijuan; Liu, Jianping; Lewith, George T. J Altern Complement Med; 16(4): 397-409, 2010 Apr.

Efficacy of acupuncture in fibromyalgia syndrome--a systematic review with a meta-analysis of controlled clinical trials. Langhorst, Jost; Klose, Petra; Musial, Frauke; Irnich, Dominik; Häuser, Winfried. Rheumatology (Oxford); 49(4): 778-88, 2010 Apr.

Traditional Chinese acupuncture and placebo (sham) acupuncture are differentiated by their effects on mu-opioid receptors (MORs). Harris, Richard E; Zubieta, Jon-Kar; Scott, David J; Napadow, Vitaly; Gracely, Richard H; Clauw, Daniel J. Neuroimage; 47(3): 1077-85, 2009 Sep.

Efectividad de la acupuntura en el alivio del dolor refractario al tratamiento farmacológico convencional / Effectiveness of acupuncture in relieving pain refractory to conventional pharmacological therapy Collazo Chao, E. Rev. Soc. Esp. Dolor; 16(2): 79-86, mar. 2009. tab, graf.

A randomized controlled trial of acupuncture added to usual treatment for fibromyalgia. Targino, Rosa Alves; Imamura, Marta; Kaziyama, Helena H S; Souza, Luiz P M; Hsing, Wu Tu; Furlan, Andréa D; Imamura, Satiko Tomikawa; Azevedo Neto, Raymundo Soares. J Rehabil Med; 40(7): 582-8, 2008 Jul.

Efeito da acupuntura na melhora da dor, sono e qualidade de vida em pacientes fibromiálgicos: estudo preliminar / Effect of acupuncture on pain, sleep and quality of life improvement in fibromyalgia patients: preliminary study Takiguchi, Raymond S; Fukuhara, Vanessa Satie; Sauer, Juliana Ferreira; Assumpção, Ana; Marques, Améilia Pasqual. Fisioter. pesqui; 15(3): 280-284, jul.-set. 2008. Tab.

Dynamic levels of glutamate within the insula are associated with improvements in multiple pain domains in fibromyalgia. Harris, Richard E; Sundgren, Pia C; Pang, Yuxi; Hsu, Michael; Petrou, Myria; Kim, Seong-Ho; McLean, Samuel A; Gracely, Richard H; Clauw, Daniel J. Arthritis Rheum; 58(3): 903-7, 2008 Mar. 


\section{Discussão}

A maior parte dos artigos que abordam a acupuntura como terapia para a fibromialgia foi publicada nos EUA, tendo o Brasil como segundo país e a China em terceiro. O fato da China ser apenas o terceiro país em número de publicações sobre o tema chama a atenção, pelo fato de a terapia ter sido criada justamente nesse país, mas deve-se observar também que a revista com maior número de artigos publicados (Zhongguo Zhen Jiu) é chinesa, o que mostra uma certa concentração de publicações no mesmo periódico, já que 66,7\% dos artigos publicados na China são da mesma revista.

Tratando do Brasil, pudemos perceber que existem artigos publicados sobre a temática aqui trabalhada, no entanto, não existe uma revista que tenha tradição para concentrar as publicações de artigos, já que nenhuma revista brasileira entrou na lista das 10 revistas com mais publicações sobre o tema.

Considerando a análise do conteúdo dos artigos, observou-se que o efeito da acupuntura é benéfico na maioria dos casos. Takiguchi et al (2008) descreveram em seu ensaio uma melhora nas variáveis sono, dor e qualidade de vida dos indivíduos estudados, porém com melhora acentuada nos indivíduos submetidos à acupuntura nos tender points. No grupo em que foi aplicada a acupuntura segundo a medicina oriental nos pontos reflexos também houve melhora, porém não foram encontradas diferenças significativas na maioria dos escores após o tratamento. Como a dor e os distúrbios do sono interferem diretamente na qualidade de vida, a melhora desses dois aspectos gerou consequente diminuição do impacto da fibromialgia na vida desses pacientes.

A qualidade do sono parece estar intimamente ligada a sintomas como a dor crônica, sendo assim o efeito analgésico obtido, aliado à liberação de substâncias semelhantes ao ópio no fluido cérebro-espinhal, sendo tais substâncias, mediadores responsáveis pelo efeito sistêmico das inserções em locais de dor, como a melhora do sono (TAKIGUCHI et al, 2008).

A diminuição do limiar de dor, mesmo em pontos onde não foi feita a acupuntura, demonstra o efeito sistêmico da inserção de agulhas, causando alívio da dor mesmo em locais distantes (TAKIGUCHI et al, 2008). A resposta inicial ocorre com estimulações da terminação nervosa sensitiva primária no interior do músculo, que envia mensagem a medula espinal e daí ao córtex (LIANZA, 2007).

Em estudo realizado por Lautenschläger et al (1989) foram aplicadas agulhas de acupuntura de duas formas distintas sendo que em um grupo as agulhas foram aplicadas sobre os tender points, e no outro grupo as agulhas foram aplicadas sobre os pontos reflexos, e a aplicação das agulhas sobre os tender points obteve resultados superiores a aplicação sobre os pontos reflexos. Já Sprott et al (1998) observou uma diminuição da concentração de serotonina nas plaquetas.

Dias et al (2012) relataram que acupuntura promove uma melhora importante na qualidade de vida de pacientes com fibromialgia. O efeito da acupuntura, de acordo com Goldenberg (2005), ocorre através da inserção das agulhas nos pontos reflexos (pontos da acupuntura) promovendo a condução de estímulos intensos e vigorosos, que tem resposta no cérebro, estimulando o sistema supressor da dor, liberando as endorfinas naturais, resultando em alívio da dor.

Segundo Goldenberg (2005) a acupuntura não tem efeito apenas sobre a dor difusa e crônica, mas também, sobre outros sintomas da fibromialgia tais como: rigidez, fadiga, sono não reparador, ansiedade e depressão, dessa forma, melhorando a qualidade de vida desses pacientes.

Sobre a literatura disponível, vale destacar a pequena quantidade de trabalhos científicos realizados sobre o uso da Acupuntura como terapia na fibromialgia. A proporção de estudos realizados ainda pode ser aumentada, visando melhor compreender os mecanismos envolvidos nas melhoras proporcionadas por tal terapia. 


\section{Conclusão}

Após a revisão dos artigos selecionados foi possível observar que acupuntura mostrouse eficaz no tratamento de indivíduos com fibromialgia, obtendo uma melhora nos aspectos referentes à qualidade de vida, dor, sono e ansiedade. No entanto, ainda se fazem necessárias mais pesquisas nessa área para maior comprovação dos efeitos da acupuntura na fibromialgia, bem como para melhor divulgar a efetividade de tal técnica.

\section{Referências}

CRISTO VV. Epidemiologia da Fibromialgia. In: Heymann et al, ed. Fibromialgia e Síndrome Miofascial. $1^{\text {a }}$ ed. São Paulo, Editora Legnar, 2006 p25-30.

DE MELO BARBOSA, Aurélio et al. Acupuntura no tratamento da fibromialgia: uma revisão de literatura. Revista Movimenta v. 6, n. 2, p. 2013.

DIAS, Marialda Höfling P. et al. Acupuntura em adolescentes com fibromialgia juvenil.Rev. paul. pediatr. São Paulo, v. 30, n. 1, 2012.

DIAS, Paulo Araujo et al. Short-term complementary and alternative medicine on quality of life in women with fibromyalgia. Journal of integrative medicine, v. 14, n. 1, p. 29-35, 2016.

GOLDENBERG, E. O coração sente, o corpodói. 3 ed, editora Atheneu, São Paulo,2005.

HEYMANN, Roberto Ezequiel et al. Consenso brasileiro do tratamento da fibromialgia. Rev bras reumatol, v. 50, n. 1, p. 56-66, 2010.

KUREBAYASHI, Leonice Fumiko Sato; FREITAS, Genival Fernandes de; OGUISSO, Taka. Enfermidades tratadas e tratáveis pela acupuntura segundo percepção de enfermeiras. Rev. esc. enferm. USP, São Paulo, v. 43, n. 4, Dec. 2009.

LIANZA, S. Medicina de Reabilitação. Editora Guanabara Koogan, Rio de Janeiro: 2007.

MARQUES, A.P. et al. Alongamento muscular em pacientes com fibromialgia a partir de um trabalho de reeducação postural global (RPG). Revista Brasileira de Reumatologia, v.34, 1994, pg. 232-234.

PROVENZA, J. R. et al. Fibromialgia. Revista Brasileira de Reumatologia, v. 44, n. 6, p. 443449, 2004.

SANTOS et. al Depressão e Qualidade de Vida em Pacientes com Fibromialgia. Revista Brasileira de fisioterapia. v. 10, n. 3, p. 317-324, 2006.

STIVAL, Rebecca Saray Marchesini et al. Acupuntura na fibromialgia: um estudo randomizadocontrolado abordando a resposta imediata da dor. Revista Brasileira de Reumatologia, v. 54, n. 6, p. 431-436, 2014.

TAKIGUCHI, Raymond S. et al. Efeito da acupuntura na melhora da dor, sono e qualidade de vida em pacientes fibromiálgicos: estudo preliminar. Fisioter. Pesqui. São Paulo , v. 15, n. 3, Sept. 2008.

WORLD HEALTH ORGANIZATION et al. Acupuncture: review and analysis of reports on controlled clinical trials. 2002. 